\title{
A deterministic tabu search algorithm for the capacitated arc routing problem
}

\author{
José Brandão ${ }^{\mathrm{a}, *}$, Richard Eglese ${ }^{\mathrm{b}}$ \\ ${ }^{a}$ Dep. de Gestão, Escola de Economia e Gestão, Universidade do Minho, Largo do Paço, 4704 -553 Braga, Portugal \\ ${ }^{\mathrm{b}}$ Department of Management Science, Lancaster University Management School, Lancaster LA1 4YX, UK
}

Available online 25 September 2006

\begin{abstract}
The capacitated arc routing problem (CARP) is a difficult optimisation problem in vehicle routing with applications where a service must be provided by a set of vehicles on specified roads. A heuristic algorithm based on tabu search is proposed and tested on various sets of benchmark instances. The computational results show that the proposed algorithm produces high quality results within a reasonable computing time. Some new best solutions are reported for a set of test problems used in the literature.
\end{abstract}

(C) 2006 Elsevier Ltd. All rights reserved.

Keywords: Heuristics; Arc routing; Tabu search

\section{Introduction}

The capacitated arc routing problem (CARP) may be described as follows: consider an undirected connected graph $G=(V, E)$, with a vertex set $V$ and edge set $E$ and a set of required edges $R \subseteq E$. A fleet of identical vehicles, each of capacity $Q$, is based at a designated depot vertex. Each edge of the graph $\left(v_{i}, v_{j}\right)$ incurs a cost $c_{i j}$ whenever a vehicle travels over it or services a required edge. When a vehicle travels over an edge without servicing it, this is referred to as deadheading. Each required edge of the graph $\left(v_{i}, v_{j}\right)$ has a demand $q_{i j}$ associated with it. A vehicle route must start and finish at the designated depot vertex and the total demand serviced on the route must not exceed the capacity of the vehicle, $Q$. The objective of the CARP is to find a minimum cost set of vehicle routes where each required edge is serviced on one of the routes.

In the instances tested, the objective is to minimise the total cost incurred on the routes and does not include any costs relating to the number of routes or vehicles required.

The graph or network on which the problem is based may be directed or undirected or mixed, but in this paper only undirected graphs are considered. A good introduction to and survey of the CARP and other arc routing problems may be found in Dror [1].

In the CARP, each edge in the graph may model a road that can be travelled in either direction and each vertex corresponds to a road junction. Applications of the CARP arise in operations such as postal deliveries, household

\footnotetext{
* Corresponding author.

E-mail addresses: sbrandao@eeg.uminho.pt (J. Brandão), R.Eglese@lancaster.ac.uk (R. Eglese).
} 
waste collection, winter gritting, snow clearance and others, though in most practical applications there are additional constraints that must also be considered, including time window constraints or restrictions on certain turns (see e.g. Lacomme et al. [2]).

The CARP is NP-hard. Even when a single vehicle is able to service all the required edges, the problem reduces to the rural postman problem (RPP) which has been shown to be NP-hard by Lenstra and Rinnooy Kan [3]. Additionally, Golden and Wong [4] showed that even 1.5-approximation for the CARP is NP-hard. Exact methods for the CARP have only been able to solve relatively small examples to optimality.

A discussion of different heuristic algorithms that have been proposed for the CARP can be found in Dror [1]. These include simple constructive heuristics (e.g. Pearn [5,6]) and go on to include various metaheuristic algorithms. The most successful algorithms that have been reported in the literature are based on different metaheuristic models. We have chosen two of these with which to compare the results of our proposed algorithm. The first is the tabu search based algorithm known as "CARPET" which is described in Hertz et al. [7]. The second is the approach using memetic algorithms, which will be referred to as MA, described in Lacomme et al. [8].

Beullens et al. [9] describe a guided local search heuristic for the CARP and also introduce some new large data sets based on the inter-city road network in Flanders (Belgium). Our proposed algorithm is also tested on these data sets.

There are also other notable contributions that have recently been proposed for solving the CARP. Greistorfer [10] also uses a tabu search based approach, but uses a form of scatter search within his proposed heuristic. Hertz and Mittaz [11] describe a variable neighbourhood descent algorithm for the CARP. Amberg et al. [12] have also proposed a tabu search based algorithm; their approach makes use of capacitated trees and can be applied to multi-depot problems. Baldacci and Maniezzo [13] describe an exact method for solving the CARP based on a new transformation to a constrained node routing problem. A similar approach is described by Longo et al. [14] who also obtain bounds and solutions to standard test problems using a transformation to the constrained vehicle routing problem.

The approach described in this paper is based on a tabu search algorithm (TSA). However, it differs from CARPET in many of the details of the implementation. In particular, the algorithm presented here is deterministic and does not require the use of random parameter values (which are also needed for MA), so making the results fully reproducible.

The structure of the remainder of this paper is as follows. Section 2 describes the methods used to obtain initial feasible solutions in our algorithm. Section 3 describes the TSA for solving the CARP and Section 4 describes the results obtained on a set of test problems. Final conclusions are presented in Section 5.

\section{Initial solutions}

Five different methods were used to obtain initial solutions. Each method provided a feasible solution that could be used as an initial solution for the TSA. The methods were designed to be fast to compute and to provide a variety of starting solutions for the TSA to improve. The diversity provided by the different starting solutions was found to be useful in ultimately finding high quality solutions.

When implementing the algorithms described in this paper, each route was represented by an ordered list of vertices, starting and ending with the depot vertex, together with a corresponding ordered list of zero-one variables, type $e_{p}$. If the $p$ th vertex in the list is $v_{i}$ and it is immediately followed by $v_{j}$, then type $e_{p}$ takes the value one if $\left(v_{i}, v_{j}\right)$ is a required edge which is serviced at this stage of the route. The cost of the route includes $c_{i j}$ and the demand satisfied by the vehicle on this route includes $q_{i j}$. If $\left(v_{i}, v_{j}\right)$ is not a required edge which is serviced at this stage of the route, then type $e_{p}$ takes the value zero, $\left(v_{i}, v_{j}\right)$ represents a path and the cost of the route is increased by the cost of deadheading this path, which is the shortest path from $v_{i}$ to $v_{j}$. This representation allows all the required operations and calculations to be carried out efficiently.

\subsection{Method 1-cheapest edge}

Each route is started with the required edge not yet served in a route that is nearest to the depot vertex. If there is more than one required edge not yet served in a route nearest to the depot vertex, then the one that has the least cost is selected. If more than one has the least cost then ties are broken arbitrarily. The vehicle starts by travelling from the depot to the nearest vertex of that edge along the least cost path (unless the first required edge is incident to the depot vertex). The route is built up by adding required edges that are feasible to the end of the route. The next edge to be added is the required edge that has not yet been included in a route which is nearest to the end vertex of the last 
included edge (and which has least cost in the event of ties, with ties broken arbitrarily if more than one edge has the same least cost), but excluding any edge that closes the tour unless no others are feasible in terms of vehicle capacity. If the next required edge is not incident to the last vertex on the route so far, the cost of the route includes the cost of deadheading along the least cost path between the last vertex and the next edge. When no remaining required edges can be feasibly added to a route, the route is completed by the vehicle returning to the depot along the least cost path from the end of the last serviced edge, and the next route is started.

\subsection{Method 2-dearest edge}

This method operates in exactly the same way as Method 1, except that the highest cost edge replaces the lowest cost edge at each selection point.

\subsection{Method 3-insert}

Each route is started as in Method 1 and a route is completed by adding the deadheading path from the end of the first edge back to the depot. Then the next edge to be chosen from the required edges not yet served and which is feasible in terms of vehicle capacity is the one that increases the cost of the route by the least amount. When considering an edge for insertion, the new edge may be inserted between any pair of required edges already included in the route that are currently linked by a deadheading path, or before the first edge or after the last edge if these edges are not directly linked to the depot.

\subsection{Method 4-connected components}

This method requires the use of a procedure to solve the RPP for sets of connected required edges. The RPP is the problem of finding the minimum cost single route to service a set of required edges in an undirected graph and in general is NP-hard [3]. However, when the subgraph generated by the subset of required edges is connected, the RPP is reduced to the undirected Chinese postman problem which is polynomially solvable [15]. A general heuristic for the RPP was first proposed in Frederickson [16], based on a procedure that is similar to Christofides' heuristic for the undirected travelling salesman problem [17]. The method has been described and used by several researchers; our version follows the version described in Pearn and Wu [18] (though in that paper it is referred to as "Christofides et al. algorithm").

Frederickson's algorithm is described in general here as it is also used within the TSA to improve individual routes. In what follows, $E_{R}=R$ for this initial solution method. However, when this algorithm is used to improve routes individually in the TSA, then $E_{R} \subseteq R$ includes only those required edges serviced by that particular route. Frederickson's algorithm may be described as follows:

1. Let $G_{R}=\left(V_{R}, E_{R}\right)$ be the subgraph of $G$ generated by $E_{R}$ with the corresponding set of vertices $V_{R}$. Let $C_{i}$ $(i=1, \ldots, c)$ be the $i$ th component $G_{R}$. Let $G_{C}$ be the graph derived from $G$ by reducing each component $C_{i}$ of $G_{R}$ to a single vertex in $G$. The cost of an edge $(i, j) \in G_{C}$ is defined as $\mathrm{d}(i, j)=\mathrm{d}\left(C_{i}, C_{j}\right)=\min _{x, y}\left\{\mathrm{~d}(x, y)-u_{x}-u_{y}\right\}$, where $x, y \in V, x \in C_{1}, y \in C_{2}$ and $u_{x}=-\eta(\operatorname{deg}(x)-2) \cdot \mathrm{d}(x, y)$ is the cost of the minimum cost path from $x$ to $y$ in the original graph $G$ and $\operatorname{deg}(x)$ is the degree of vertex $x$ in the original graph $G$.

2. Determine the minimum cost spanning tree in $G_{C}$. Let $E_{\mathrm{T}}$ be the set of edges from the minimum cost spanning tree solution.

3. Considering the graph $G_{R} \cup E_{\mathrm{T}}$ find a minimum cost perfect matching of the odd degree vertices. Let $E_{\mathrm{M}}$ be the edges of the matching.

4. Find an Eulerian tour in the graph $G_{R} \cup E_{\mathrm{T}} \cup E_{\mathrm{M}}$. This tour is the RPP solution.

Pearn and $\mathrm{Wu}$ show how the results can be influenced by the use of a parameter $\eta$. In our implementation, the algorithm is executed with $\eta=0$, then $\eta=1$ and the better solution is chosen.

Note that Frederickson's heuristic includes finding a minimum cost perfect matching as one of the steps. This may be achieved optimally in polynomial time using the approach described in Edmonds and Johnson [15]. However, this 
complex algorithm was not available and so a simple greedy heuristic was used for the matching step, which can be described as follows:

Step 0: Set every vertex as non-included.

Step 1: Select the edge with the minimum cost among all the edges of the graph, linking two non-included vertices. Set the two end vertices of this edge as included.

Step 2: Repeat step 1 until all the vertices are included.

If the number of vertices were less than or equal to six, then the heuristic was not applied and the optimum solution was found by complete enumeration. Tests were carried out to see what effect the use of this procedure had on the solution to the CARP by finding the optimum solution to the matching problem by complete enumeration in all cases. As expected, the complete enumeration method was much slower than the procedure using the heuristic, but surprisingly, the average solutions to the CARP were worse than when the heuristic was used. We therefore retained the heuristic procedure for solving the matching problem.

Thus for each connected component, a route is constructed using Frederickson's heuristic, ignoring the capacity constraints. In the special case where more than two required edges are adjacent to the depot vertex, the connected component is split again into connected components in which no more than two required edges connect to the depot. This is done by splitting the route into subroutes: following the order of the route it is cut at the point where the second edge linked to the depot appears; then a new route starts and this process finishes when only one or two edges linked to the depot remain.

The route is modified into one starting and ending at the depot by starting with a required edge connected to the depot if one exists, otherwise adding the least cost route from the depot to a required edge in the connected component, following the constructed route until the last required edge in the connected component has been included and then finally joining the route to the depot by linking back to the depot along the least cost path. If any route is infeasible according to the capacity constraint, it is split into smaller routes in the following way. Starting from the depot, the route is followed until the last feasible required edge has been included. The route is then completed by adding the least cost path from the end of this edge back to the depot. The next route is started by adding the least cost path from the depot to the next required edge that is to be served on the route that had been created. The process finishes when all required edges in each connected component have been included in a route.

\subsection{Method 5-path scanning}

This method is based on the procedure proposed by Golden et al. [19]. Each route is extended by one required edge at each step using a variety of selection rules.

Each route starts at the depot vertex. Let $S$ be the set of required edges closest to the end of the current route that are not yet served and do not exceed the capacity of the current route. If $S$ is empty then complete the current route using the shortest deadheading path from the end of the current route to the depot vertex and start a new route. If $S$ is not empty, exclude from $S$ any edges that would close the route unless that would make $S$ empty. Select a required edge in $S$ to be the next edge in the route to be serviced according to the current rule and extend the current route to the vertex at the end of the selected edge.

Five rules are used to determine the next required edge, $e$, in the route to be serviced: (1) maximise the distance to the depot; (2) minimise the distance to the depot; (3) use rule 1 if the vehicle is less than half-full, else use rule 2; (4) maximise the ratio $d(e) / c(e)$, where $d(e)$ and $c(e)$ are, respectively, the demand and the cost of $e$; (5) minimise this ratio. Each criterion gives rise to one solution and the best of the five is chosen.

Some results to show the effectiveness of these initial solution methods are given in Section 4.

\section{The tabu search algorithm}

\subsection{Neighbourhood moves}

The TSA is based on three types of neighbourhood move. The first two are insertions (single and double) and the third is a swap.

In a single insertion move, a candidate edge (only required edges can be candidates) is removed from its current route and a trial insertion is made in any other route between any two serviced edges that are not adjacent, but are 
linked by a deadheading path of edges. The trial insertion considers both directions for the edge to be traversed when inserted in the new route. In a double insertion move, the operation is similar except that a candidate consists of two connected required edges in one route.

The swap move is similar. Two candidate edges are selected from two different routes, each containing deadheading paths. The candidate edges are removed from their original routes and inserted in the other routes between any two serviced edges that are not adjacent, but are linked by a deadheading path of edges.

In this implementation, the frequencies of the different types of move may change according to the phase of the algorithm. Parameters, $F_{\mathrm{SI}}, F_{\mathrm{DI}}$ and $F_{\mathrm{SWAP}}$ denote the frequencies of the single insertion, double insertion and swap

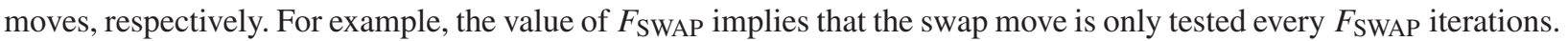

The trial move chosen depends on the effect of the move on the objective function defined in the next section.

\subsection{Objective function}

The objective function to be minimised by the TSA includes the total cost of each route, $i$, plus a penalty cost $P w(i)$, where $P$ is a penalty term and $w(i)=\max (x(i)-Q, 0)$, and where $x(i)$ is the sum of the demands on the edges serviced in route $i$. For any candidate solution, $S$, the objective function is denoted by $f(S)$.

The parameter $P$ is set at 1 initially, and is then halved if all the solutions are feasible for 10 consecutive iterations; it is doubled if all the solutions are infeasible for 10 consecutive iterations. A similar use of $P$ to direct the search from feasible to infeasible regions and vice versa has also been used before by e.g. Hertz et al. [7] and Beullens et al. [9].

\subsection{Admissibility of moves}

A conventional tabu list is constructed to prevent the reversal of accepted moves for the next $t$ iterations, where $t$ is the length of the tabu list. The length of the tabu list is fixed and after some experimentation has been set to $N / 2$ in Phase 1 of the TSA and $N / 6$ in Phase 2, where $N$ is the number of required edges. The tabu restriction may be overridden if the move will produce a solution that is better than what has been found in the past. This is referred to as the aspiration criterion.

In the TSA, a trial move to solution $S^{\prime}$ is regarded as admissible:

(i) if it is not currently on the tabu list;

(ii) or if it is tabu and infeasible, but the value of $f\left(S^{\prime}\right)$ is less than the value of the best infeasible solution yet found;

(iii) or if it is tabu and feasible, but the value of $f\left(S^{\prime}\right)$ is less than the value of the best feasible solution yet found.

\subsection{Route improvement procedure}

At the end of each iteration, a procedure is applied to each of the two individual routes that have been changed to try to reduce their costs further. For each individual route we aim to find the least cost route, starting from the depot and then serving all the required edges included for service in the route and returning to the depot. This is done by using Frederickson's heuristic, which was described in Section 2.4.

\subsection{Outline of TSA}

The operation of the TSA can now be summarised in the following steps:

\section{Initialise:}

Set current solution $S$ to be an initial solution and let $f(S)$ be the total cost of $S$

Set best solution $S_{\mathrm{B}}=S$

Set best feasible solution $S_{\mathrm{BF}}=S$, if $S$ is feasible; otherwise set $f\left(S_{\mathrm{BF}}\right)=\infty$

Set iteration counter, $k=0$

Set number of iterations since best solution after Intensification step, $k_{\mathrm{B}}=0$ 
Set number of iterations for applying Intensification step, $k_{L}=8 \mathrm{~N}$

Set number of iterations since best feasible solution, $k_{\mathrm{BF}}=0$

Set number of consecutive iterations that solution is feasible, $k_{\mathrm{F}}=0$

Set number of consecutive iterations that solution is infeasible, $k_{\mathrm{I}}=0$

Set number of iterations since best solution in total, $k_{\mathrm{BT}}=0$

Set tabu list to be empty

Set tabu tenure, $t=N / 2$

Set frequency parameters, $F_{\mathrm{SI}}=1, F_{\mathrm{DI}}=5, F_{\mathrm{SWAP}}=5$

Set penalty parameter, $P=1$.

2. Find neighbourhood move $\left(S^{\prime}\right)$ from $S$, using all required edges as a candidate list:

Set $f\left(S^{\prime}\right)=\infty$.

2.1. If $k$ is a multiple of $F_{\mathrm{SI}}$ perform trial single insertion moves

For each admissible move, $s$, do:

If $f(s)<f\left(S^{\prime}\right)$, then

(1) $S^{\prime}=s$ and $f\left(S^{\prime}\right)=f(s)$;

(2) If $s$ is feasible and $f(s)<f\left(S_{\mathrm{BF}}\right)$, or $f(s)<f\left(S_{\mathrm{B}}\right)$ go to Step 3.

Repeat until all the potential moves have been explored.

2.2. If $k$ is a multiple of $F_{\mathrm{DI}}$ perform trial double insertion moves

For each admissible move, $s$, do:

If $f(s)<f\left(s^{\prime}\right)$, then

(1) $S^{\prime}=s$ and $f\left(S^{\prime}\right)=f(s)$;

(2) If $s$ is feasible and $f(s)<f\left(S_{\mathrm{BF}}\right)$, or $f(s)<f\left(S_{\mathrm{B}}\right)$ go to Step 3.

Repeat until all the potential moves have been explored.

2.3. If $k$ is a multiple of $F_{\mathrm{SWAP}}$ perform trial swap moves

For each admissible move, $s$, do:

If $f(s)<f\left(S^{\prime}\right)$, then

(1) $S^{\prime}=s$ and $f\left(S^{\prime}\right)=f(s)$;

(2) If $s$ is feasible and $f(s)<f\left(S_{\mathrm{BF}}\right)$, or $f(s)<f\left(S_{\mathrm{B}}\right)$ go to Step 3.

Repeat until all the potential moves have been explored.

3. Improve changed routes:

For each of the two routes that have been changed, apply the route improvement procedure using Frederickson's heuristic to try to find further cost reductions if possible, resulting in solution $S^{\prime \prime}$.

4. Update:

Update tabu list.

If $S^{\prime \prime}$ is feasible and $f\left(S^{\prime \prime}\right)<f\left(S_{\mathrm{BF}}\right)$ then

(1) apply Frederickson's heuristic to each route individually (except the two routes that have just been updated in Step 3) to get a new solution $S^{\prime \prime \prime}$

(2) set $S_{\mathrm{BF}}=S^{\prime \prime \prime}, S^{\prime \prime}=S^{\prime \prime \prime}$ and set $k_{\mathrm{B}}=k_{\mathrm{BF}}=k_{\mathrm{BT}}=0$.

If $f\left(S^{\prime \prime}\right)<f\left(S_{\mathrm{B}}\right)$ then set $S_{\mathrm{B}}=S^{\prime \prime}$ and $k_{\mathrm{B}}=k_{\mathrm{BT}}=0$.

Set $k=k+1, k_{\mathrm{B}}=k_{\mathrm{B}}+1, k_{\mathrm{BF}}=k_{\mathrm{BF}}+1, k_{\mathrm{BT}}=k_{\mathrm{BT}}+1$.

If $k$ is a multiple of 10 , then if $k_{\mathrm{F}}=10$, then set $P=P / 2$ else if $k_{\mathrm{I}}=10$ then set $P=2 P$;

If $k_{\mathrm{F}}=10$ or $k_{\mathrm{I}}=10$ then recalculate $f\left(S_{\mathrm{B}}\right)$ with the new value of $P$ and set $k_{\mathrm{F}}=k_{\mathrm{I}}=0$.

5. Change of parameter values:

If $k_{\mathrm{B}}=k_{L} / 2$, then set $F_{\mathrm{SI}}=5, F_{\mathrm{DI}}=1, F_{\mathrm{SWAP}}=10$.

6. Intensification:

If $k_{\mathrm{B}}=k_{\mathrm{L}}$, then

(1) if $f\left(S_{\mathrm{BF}}\right)<\infty$ then set $S=S_{\mathrm{BF}}$ else set $S=S_{\mathrm{B}}$,

(2) set $k_{\mathrm{B}}=0, P=1, k_{\mathrm{F}}=0, k_{\mathrm{I}}=0, F_{\mathrm{SI}}=1, F_{\mathrm{DI}}=5, F_{\mathrm{SWAP}}=5, k_{\mathrm{L}}=k_{\mathrm{L}}+2 N$, 
Table 1

Ratios of average solution values to average best solutions for the initial solution methods

\begin{tabular}{llllll}
\hline & Cheapest & Dearest & Insert & Connected components & Path scanning \\
\hline DeArmon & 1.25 & 1.25 & 1.19 & 1.29 & 1.13 \\
Benavent et al. & 1.27 & 1.25 & 1.28 & 1.25 & 1.19 \\
Eglese & 1.33 & 1.26 & 1.29 & 1.24 & 1.16 \\
\hline
\end{tabular}

Table 2

Problems from DeArmon [20]

\begin{tabular}{|c|c|c|c|c|c|c|c|c|c|c|c|c|}
\hline \multirow[t]{2}{*}{ No. } & \multirow[t]{2}{*}{$|V|$} & \multirow[t]{2}{*}{$|E|$} & \multirow[t]{2}{*}{ Best known } & \multirow[t]{2}{*}{ Our best } & \multicolumn{2}{|c|}{ CARPET } & \multicolumn{2}{|l|}{ MA } & \multicolumn{2}{|c|}{ TSA Version 1} & \multicolumn{2}{|c|}{ TSA Version 2} \\
\hline & & & & & Cost & $\operatorname{CPU}(\mathrm{s})^{\mathrm{a}}$ & Cost & $\mathrm{CPU}(\mathrm{s})^{\mathrm{b}}$ & Cost & $\operatorname{CPU}(\mathrm{s})^{\mathrm{c}}$ & Cost & $\operatorname{CPU}(s)^{\mathrm{c}}$ \\
\hline 1 & 12 & 22 & 316 & 316 & 316 & 2.4 & 316 & 0.0 & 316 & 0.0 & 316 & 0.0 \\
\hline 2 & 12 & 26 & 339 & 339 & 339 & 4.0 & 339 & 0.3 & 345 & 0.1 & 339 & 0.1 \\
\hline 3 & 12 & 22 & 275 & 275 & 275 & 0.1 & 275 & 0.0 & 275 & 0.0 & 275 & 0.0 \\
\hline 4 & 11 & 19 & 287 & 287 & 287 & 0.1 & 287 & 0.0 & 287 & 0.0 & 287 & 0.0 \\
\hline 5 & 13 & 26 & 377 & 377 & 377 & 4.3 & 377 & 0.1 & 377 & 0.0 & 377 & 0.1 \\
\hline 6 & 12 & 22 & 298 & 298 & 298 & 0.7 & 298 & 0.1 & 298 & 0.0 & 298 & 0.0 \\
\hline 7 & 12 & 22 & 325 & 325 & 325 & 0.0 & 325 & 0.1 & 325 & 0.0 & 325 & 0.0 \\
\hline 10 & 27 & 46 & 348 & 348 & 352 & 47.2 & 350 & 26.5 & 352 & 2.5 & 348 & 1.6 \\
\hline 11 & 27 & 51 & 303 & 303 & 317 & 41.8 & 303 & 4.7 & 307 & 1.0 & 303 & 26.1 \\
\hline 12 & 12 & 25 & 275 & 275 & 275 & 1.2 & 275 & 0.1 & 275 & 0.0 & 275 & 0.0 \\
\hline 13 & 22 & 45 & 395 & 395 & 395 & 1.8 & 395 & 0.9 & 395 & 0.2 & 395 & 0.1 \\
\hline 14 & 13 & 23 & 458 & 458 & 458 & 16.0 & 458 & 6.5 & 462 & 0.2 & 458 & 0.8 \\
\hline 15 & 10 & 28 & 536 & 536 & 544 & 1.9 & 536 & 4.9 & 544 & 0.2 & 540 & 4.8 \\
\hline 16 & 7 & 21 & 100 & 100 & 100 & 0.4 & 100 & 0.1 & 100 & 0.0 & 100 & 0.1 \\
\hline 17 & 7 & 21 & 58 & 58 & 58 & 0.0 & 58 & 0.0 & 58 & 0.0 & 58 & 0.0 \\
\hline 18 & 8 & 28 & 127 & 127 & 127 & 1.3 & 127 & 0.1 & 129 & 0.2 & 127 & 0.1 \\
\hline 19 & 8 & 28 & 91 & 91 & 91 & 0.0 & 91 & 0.1 & 91 & 0.0 & 91 & 0.0 \\
\hline 20 & 9 & 36 & 164 & 164 & 164 & 0.2 & 164 & 0.1 & 164 & 0.0 & 164 & 0.0 \\
\hline 21 & 8 & 11 & 55 & 55 & 55 & 0.2 & 55 & 0.0 & 55 & 0.0 & 55 & 0.0 \\
\hline 22 & 11 & 22 & 121 & 121 & 121 & 7.4 & 121 & 0.2 & 123 & 0.1 & 121 & 0.2 \\
\hline 23 & 11 & 33 & 156 & 156 & 156 & 0.9 & 156 & 0.1 & 156 & 0.0 & 156 & 0.0 \\
\hline 24 & 11 & 44 & 200 & 200 & 200 & 2.6 & 200 & 2.3 & 200 & 0.0 & 200 & 0.1 \\
\hline 25 & 11 & 55 & 233 & 233 & 235 & 26.6 & 233 & 34.1 & 235 & 0.7 & 235 & 22.3 \\
\hline \multicolumn{3}{|l|}{ Average } & 253.8 & 253.8 & 255.0 & 7.0 & 253.9 & 3.5 & 255.2 & 0.2 & 254.0 & 2.5 \\
\hline \multicolumn{3}{|l|}{ No. optimal } & 23 & 23 & 19 & - & 22 & - & 15 & - & 21 & - \\
\hline \multicolumn{3}{|c|}{$\begin{array}{l}\text { Average deviation from } \\
\text { Best known }(\%)\end{array}$} & & 0.00 & 0.47 & - & 0.04 & - & 0.55 & - & 0.08 & - \\
\hline
\end{tabular}

Optimal solutions are in bold.

${ }^{a}$ The original value has been divided by 7 (SGI Indigo2 at $195 \mathrm{MHz}$ ).

${ }^{\mathrm{b}}$ The original value has been divided by 1.5 (Pentium III at $1 \mathrm{GHz}$ ).

${ }^{\mathrm{c}}$ We used a Pentium Mobile at $1.4 \mathrm{GHz}$.

(3) recalculate $f\left(S_{\mathrm{B}}\right)$ with the new value of $P$, and empty tabu list.

(Note that the changes to the parameter values and emptying the tabu list at this point means that the sequence of solutions following $S$ is different to the sequence generated previously.)

\section{Stopping criterion:}

If $\left(k \geqslant 900\lceil\sqrt{ } N\rceil\right.$ and $\left.k_{\mathrm{BF}} \geqslant 10 N\right)$ or $k_{\mathrm{BT}}=2 k_{L}$ then stop, otherwise go to Step 2.

Two versions of the TSA have been applied to the experimental set of problems. In Version 1, the initial solution is taken from the path scanning method as described in Section 2.5, because this method gave the best results on average for the initial solution methods tested. This provides a good initial solution quickly. 
Table 3

Problems from Benavent et al. [21]

\begin{tabular}{|c|c|c|c|c|c|c|c|c|c|c|c|c|c|}
\hline \multirow[t]{2}{*}{ Name } & \multirow[t]{2}{*}{$|V|$} & \multirow[t]{2}{*}{$|E|$} & \multirow[t]{2}{*}{ Lower bound } & \multirow[t]{2}{*}{ Best known } & \multirow[t]{2}{*}{ Our best } & \multicolumn{2}{|c|}{ CARPET } & \multicolumn{2}{|l|}{ MA } & \multicolumn{2}{|c|}{ TSA Version 1} & \multicolumn{2}{|c|}{ TSA Version 2} \\
\hline & & & & & & Cost & CPU (s) & Cost & CPU (s) & Cost & CPU (s) & Cost & $\mathrm{CPU}(\mathrm{s})$ \\
\hline $1 \mathrm{~A}$ & 24 & 39 & 173 & 173 & 173 & 173 & 0.0 & 173 & 0.0 & 173 & 0.0 & 173 & 0.0 \\
\hline $1 \mathrm{~B}$ & 24 & 39 & 173 & 173 & 173 & 173 & 7.2 & 173 & 5.3 & 173 & 0.2 & 173 & 0.9 \\
\hline $1 \mathrm{C}$ & 24 & 39 & 245 & 245 & 245 & 245 & 72.3 & 245 & 19.1 & 245 & 0.8 & 245 & 12.1 \\
\hline $2 \mathrm{~A}$ & 24 & 34 & 227 & 227 & 227 & 227 & 0.1 & 227 & 0.1 & 227 & 0.0 & 227 & 0.0 \\
\hline $2 \mathrm{~B}$ & 24 & 34 & 259 & 259 & 259 & 260 & 10.1 & 259 & 0.1 & 259 & 0.1 & 259 & 0.3 \\
\hline $2 \mathrm{C}$ & 24 & 34 & 457 & 457 & 457 & 494 & 24.5 & 457 & 14.5 & 457 & 1.5 & 457 & 7.8 \\
\hline $3 \mathrm{~A}$ & 24 & 35 & 81 & 81 & 81 & 81 & 0.6 & 81 & 0.1 & 81 & 0.0 & 81 & 0.0 \\
\hline $3 \mathrm{~B}$ & 24 & 35 & 87 & 87 & 87 & 87 & 2.1 & 87 & 0.0 & 87 & 0.0 & 87 & 0.0 \\
\hline $3 \mathrm{C}$ & 24 & 35 & 138 & 138 & 138 & 138 & 32.2 & 138 & 18.8 & 138 & 0.6 & 138 & 1.3 \\
\hline $4 \mathrm{~A}$ & 41 & 69 & 400 & 400 & 400 & 400 & 21.9 & 400 & 0.5 & 400 & 0.1 & 400 & 0.4 \\
\hline $4 B$ & 41 & 69 & 412 & 412 & 412 & 416 & 58.6 & 412 & 0.8 & 414 & 1.7 & 412 & 5.5 \\
\hline $4 C$ & 41 & 69 & 428 & 428 & 428 & 453 & 54.2 & 428 & 12.7 & 444 & 1.7 & 428 & 38.0 \\
\hline $4 \mathrm{D}$ & 41 & 69 & 526 & 530 & 530 & 556 & 180.8 & 541 & 68.9 & 538 & 10.3 & 530 & 110.0 \\
\hline $5 \mathrm{~A}$ & 34 & 65 & 423 & 423 & 423 & 423 & 2.9 & 423 & 1.3 & 423 & 0.3 & 423 & 0.3 \\
\hline $5 B$ & 34 & 65 & 446 & 446 & 446 & 448 & 32.0 & 446 & 0.7 & 446 & 0.1 & 446 & 0.1 \\
\hline $5 \mathrm{C}$ & 34 & 65 & 473 & 474 & 474 & 476 & 41.3 & 474 & 67.3 & 474 & 1.2 & 474 & 10.6 \\
\hline $5 \mathrm{D}$ & 34 & 65 & 573 & 579 & 577 & 607 & 173.5 & 583 & 60.5 & 583 & 6.5 & 583 & 73.3 \\
\hline $6 \mathrm{~A}$ & 31 & 50 & 223 & 223 & 223 & 223 & 3.0 & 223 & 0.1 & 223 & 0.1 & 223 & 1.6 \\
\hline $6 \mathrm{~B}$ & 31 & 50 & 233 & 233 & 233 & 241 & 20.9 & 233 & 44.9 & 233 & 2.5 & 233 & 12.7 \\
\hline $6 \mathrm{C}$ & 31 & 50 & 317 & 317 & 317 & 329 & 66.0 & 317 & 34.8 & 323 & 3.1 & 317 & 22.9 \\
\hline $7 \mathrm{~A}$ & 40 & 66 & 279 & 279 & 279 & 279 & 5.1 & 279 & 1.3 & 283 & 0.9 & 279 & 1.0 \\
\hline $7 \mathrm{~B}$ & 40 & 66 & 283 & 283 & 283 & 283 & 0.0 & 283 & 0.3 & 283 & 0.1 & 283 & 0.5 \\
\hline $7 \mathrm{C}$ & 40 & 66 & 334 & 334 & 334 & 343 & 94.0 & 334 & 67.5 & 335 & 4.0 & 334 & 37.0 \\
\hline $8 \mathrm{~A}$ & 30 & 63 & 386 & 386 & 386 & 386 & 3.0 & 386 & 0.5 & 386 & 0.6 & 386 & 0.3 \\
\hline $8 B$ & 30 & 63 & 395 & 395 & 395 & 401 & 63.1 & 395 & 6.7 & 407 & 1.0 & 395 & 1.8 \\
\hline $8 \mathrm{C}$ & 30 & 63 & 518 & 521 & 521 & 533 & 114.1 & 527 & 47.7 & 545 & 1.9 & 529 & 55.7 \\
\hline $9 \mathrm{~A}$ & 50 & 92 & 323 & 323 & 323 & 323 & 22.1 & 323 & 12.2 & 323 & 0.7 & 323 & 0.0 \\
\hline $9 \mathrm{~B}$ & 50 & 92 & 326 & 326 & 326 & 329 & 46.4 & 326 & 19.6 & 326 & 1.3 & 326 & 0.5 \\
\hline $9 \mathrm{C}$ & 50 & 92 & 332 & 332 & 332 & 332 & 43.7 & 332 & 47.5 & 332 & 0.7 & 332 & 0.4 \\
\hline $9 \mathrm{D}$ & 50 & 92 & 385 & 391 & 391 & 409 & 273.5 & 391 & 140.7 & 404 & 7.3 & 391 & 60.4 \\
\hline $10 \mathrm{~A}$ & 50 & 97 & 428 & 428 & 428 & 428 & 4.3 & 428 & 17.0 & 430 & 3.5 & 428 & 3.2 \\
\hline 10B & 50 & 97 & 436 & 436 & 436 & 436 & 14.3 & 436 & 3.1 & 438 & 3.6 & 436 & 1.8 \\
\hline $10 \mathrm{C}$ & 50 & 97 & 446 & 446 & 446 & 451 & 72.4 & 446 & 11.5 & 447 & 4.6 & 446 & 7.5 \\
\hline $10 \mathrm{D}$ & 50 & 97 & 525 & 526 & 528 & 544 & 121.0 & 530 & 143.3 & 534 & 10.9 & 530 & 218.1 \\
\hline Average & & & & 344.4 & 344.4 & 350.8 & 49.5 & 345.2 & 25.6 & 347.5 & 2.1 & 344.9 & 20.2 \\
\hline No. optimal & & & & 28 & 28 & 16 & - & 23 & - & 16 & - & 23 & - \\
\hline No. best & & & & 33 & 33 & 17 & - & 30 & - & 20 & - & 31 & - \\
\hline $\begin{array}{l}\text { Average devia } \\
\text { from Best kno }\end{array}$ & $\begin{array}{l}\text { tion } \\
\text { own }(c\end{array}$ & & & & 0 & 1.86 & - & 0.23 & - & 0.90 & - & 0.15 & - \\
\hline
\end{tabular}

Optimal solutions are in bold.

In Version 2, the TSA works in four phases. In Phase 1, the TSA is applied to each of the five initial solutions described in Section 2. In Phase 2, the TSA is applied once more to the best solution from Phase 1, but with $t=N / 6$ and initial values of $F_{\mathrm{SI}}=1, F_{\mathrm{DI}}=10, F_{\mathrm{SWAP}}=3$. Phase 3 works as Phase 1 , but with $t=N / 8$ and fixed values of $F_{\mathrm{SI}}=1, F_{\mathrm{DI}}=10, F_{\mathrm{SWAP}}=\infty$, i.e. $F_{\mathrm{SI}}, F_{\mathrm{DI}}, F_{\mathrm{SWAP}}$ are not updated in Step 5 or Step $6(2)$. This is followed by applying 
Table 4

Problems from Eglese

\begin{tabular}{|c|c|c|c|c|c|c|c|c|c|c|c|c|}
\hline \multirow[t]{2}{*}{ Name } & \multirow[t]{2}{*}{$|V|$} & \multirow[t]{2}{*}{$|R|$} & \multirow[t]{2}{*}{$|E|$} & \multirow[t]{2}{*}{ LB } & \multirow[t]{2}{*}{ Best known } & \multirow[t]{2}{*}{ Our best } & \multicolumn{2}{|l|}{ MA } & \multicolumn{2}{|c|}{ TSA Version 1} & \multicolumn{2}{|c|}{ TSA Version 2} \\
\hline & & & & & & & Cost & CPU (s) & Cost & CPU (s) & Cost & CPU (s) \\
\hline E1-A & 77 & 51 & 98 & 3548 & 3548 & 3548 & 3548 & 49.5 & 3548 & 2.1 & 3548 & 22.1 \\
\hline E1-B & 77 & 51 & 98 & 4498 & 4498 & 4498 & 4498 & 46.3 & 4533 & 4.8 & 4533 & 28.0 \\
\hline E1-C & 77 & 51 & 98 & 5566 & 5595 & 5595 & 5595 & 47.5 & 5659 & 5.1 & 5595 & 24.1 \\
\hline E2-A & 77 & 72 & 98 & 5018 & 5018 & 5018 & 5018 & 101.7 & 5018 & 7.7 & 5018 & 63.4 \\
\hline E2-B & 77 & 72 & 98 & 6305 & 6340 & 6317 & 6340 & 102.3 & 6385 & 11.5 & 6343 & 66.7 \\
\hline $\mathrm{E} 2-\mathrm{C}$ & 77 & 72 & 98 & 8243 & 8395 & 8335 & 8415 & 86.4 & 8400 & 12.0 & 8347 & 78.7 \\
\hline E3-A & 77 & 87 & 98 & 5898 & 5898 & 5898 & 5898 & 161.3 & 6044 & 17.9 & 5902 & 77.3 \\
\hline E3-B & 77 & 87 & 98 & 7704 & 7816 & 7777 & 7822 & 170.2 & 7916 & 17.8 & 7816 & 113.4 \\
\hline E3-C & 77 & 87 & 98 & 10163 & 10369 & 10305 & 10433 & 137.6 & 10309 & 23.2 & 10309 & 134.3 \\
\hline E4-A & 77 & 98 & 98 & 6408 & 6461 & 6456 & 6461 & 194.6 & 6476 & 14.0 & 6473 & 135.5 \\
\hline E4-B & 77 & 98 & 98 & 8884 & 9021 & 9000 & 9021 & 208.6 & 9134 & 26.9 & 9063 & 167.6 \\
\hline E4-C & 77 & 98 & 98 & 11427 & 11779 & 11601 & 11779 & 168.3 & 11627 & 31.8 & 11627 & 188.6 \\
\hline S1-A & 140 & 75 & 190 & 5018 & 5018 & 5018 & 5018 & 139.1 & 5171 & 10.3 & 5072 & 66.6 \\
\hline S1-B & 140 & 75 & 190 & 6384 & 6435 & 6388 & 6435 & 139.2 & 6388 & 13.1 & 6388 & 80.8 \\
\hline S1-C & 140 & 75 & 190 & 8493 & 8518 & 8518 & 8518 & 110.4 & 8739 & 6.9 & 8535 & 79.2 \\
\hline S2-A & 140 & 147 & 190 & 9824 & 9995 & 9956 & 9995 & 582.9 & 10190 & 70.2 & 10038 & 395.1 \\
\hline S2-B & 140 & 147 & 190 & 12968 & 13174 & 13165 & 13174 & 507.0 & 13284 & 78.2 & 13178 & 448.3 \\
\hline $\mathrm{S} 2-\mathrm{C}$ & 140 & 147 & 190 & 16353 & 16715 & 16505 & 16795 & 498.0 & 16709 & 53.6 & 16505 & 515.8 \\
\hline S3-A & 140 & 159 & 190 & 10143 & 10296 & 10260 & 10296 & 713.7 & 10508 & 79.3 & 10451 & 554.2 \\
\hline S3-B & 140 & 159 & 190 & 13616 & 14028 & 13807 & 14053 & 709.3 & 13981 & 84.2 & 13981 & 570.6 \\
\hline $\mathrm{S} 3-\mathrm{C}$ & 140 & 159 & 190 & 17100 & 17297 & 17234 & 17297 & 582.9 & 17346 & 99.1 & 17346 & 596.4 \\
\hline S4-A & 140 & 190 & 190 & 12143 & 12442 & 12341 & 12442 & 1025.1 & 12546 & 129.8 & 12462 & 696.8 \\
\hline S4-B & 140 & 190 & 190 & 16093 & 16531 & 16442 & 16531 & 953.5 & 16695 & 141.4 & 16490 & 954.6 \\
\hline S4-C & 140 & 190 & 190 & 20375 & 20832 & 20591 & 20832 & 996.7 & 20981 & 144.9 & 20733 & 934.5 \\
\hline Average & & & & 9673.8 & 9834.1 & 9773.9 & 9842.3 & 351.4 & 9899.5 & 45.2 & 9823.0 & 291.4 \\
\hline No. optimal & & & & & 5 & 5 & 5 & - & 2 & - & 2 & - \\
\hline No. best & & & & - & 7 & 24 & 7 & - & 3 & - & 5 & - \\
\hline $\begin{array}{l}\text { Average devi } \\
\text { to the LB }(\%)\end{array}$ & tion & & & - & 1.66 & 1.03 & 1.74 & - & 2.33 & - & 1.54 & - \\
\hline
\end{tabular}

Optimal solutions are in bold.

New best solutions are in italic.

Phase 2 again to the best solution just found. Finally, Phase 4 applies the TSA to the best solution of the previous phases with the following parameters: $t=N / 3, k_{\mathrm{B}}=25 N$, and fixed values of $F_{\mathrm{SI}}=1, F_{\mathrm{DI}}=10, F_{\mathrm{SWAP}}=3$. Phases 3 and 4 were not applied to the Eglese set of problems, as for these larger problems, the additional computing time required gave only a small improvement.

\section{Results of experiments}

Computational experiments have initially been conducted on three sets of CARP problems that have been studied in the literature. The first set contains 23 instances originally generated by DeArmon [20] and discussed by Golden et al. [19]. The second set is from Benavent et al. [21] containing 34 instances defined on 10 different graphs; for each graph different instances were created by changing the capacity of the vehicles. In both the first two sets of problems, all the edges in the graphs are required edges. The final set was generated by Eglese based on data from a winter gritting 
Table 5

Problems from Beullens et al.- set C

\begin{tabular}{|c|c|c|c|c|c|c|c|c|c|c|c|}
\hline \multirow[t]{2}{*}{ Name } & \multirow[t]{2}{*}{$|V|$} & \multirow[t]{2}{*}{$|R|$} & \multirow[t]{2}{*}{$|E|$} & \multirow[t]{2}{*}{ LB } & \multirow[t]{2}{*}{ Our best } & \multicolumn{2}{|c|}{ Beullens et al. } & \multicolumn{2}{|c|}{ TSA Version 1} & \multicolumn{2}{|c|}{ TSA Version 2} \\
\hline & & & & & & Cost & $\operatorname{CPU}(\mathrm{s})^{\mathrm{a}}$ & Cost & CPU (s) & Cost & CPU (s) \\
\hline $\mathrm{C} 1$ & 69 & 79 & 98 & 1590 & 1660 & 1660 & 325.4 & 1700 & 12.7 & 1660 & 127.1 \\
\hline $\mathrm{C} 2$ & 48 & 53 & 66 & 1095 & 1095 & 1095 & 7.9 & 1165 & 1.6 & 1095 & 26.6 \\
\hline $\mathrm{C} 3$ & 46 & 51 & 64 & 875 & 925 & 925 & 172.6 & 935 & 3.8 & 925 & 19.4 \\
\hline $\mathrm{C} 4$ & 60 & 72 & 84 & 1285 & 1340 & 1340 & 283.7 & 1515 & 11.0 & 1340 & 65.4 \\
\hline $\mathrm{C} 5$ & 56 & 65 & 79 & 2410 & 2470 & 2475 & 234.3 & 2630 & 6.8 & 2470 & 47.8 \\
\hline C6 & 38 & 51 & 55 & 855 & 895 & 895 & 160.4 & 910 & 2.7 & 895 & 9.3 \\
\hline $\mathrm{C} 7$ & 54 & 52 & 70 & 1735 & 1795 & 1795 & 166.6 & 1795 & 5.4 & 1795 & 29.9 \\
\hline $\mathrm{C} 8$ & 66 & 63 & 88 & 1640 & 1730 & 1730 & 229.8 & 1740 & 7.2 & 1730 & 44.4 \\
\hline C9 & 76 & 97 & 117 & 1775 & 1820 & 1825 & 445.1 & 1880 & 24.6 & 1830 & 245.9 \\
\hline $\mathrm{C} 10$ & 60 & 55 & 82 & 2190 & 2270 & 2290 & 181.1 & 2370 & 5.5 & 2270 & 30.6 \\
\hline $\mathrm{C} 11$ & 83 & 94 & 118 & 1725 & 1815 & 1815 & 423.6 & 1940 & 22.8 & 1815 & 209.4 \\
\hline C12 & 62 & 72 & 88 & 1510 & 1610 & 1610 & 285.2 & 1760 & 10.8 & 1610 & 46.2 \\
\hline C13 & 40 & 52 & 60 & 1050 & 1110 & 1110 & 173.6 & 1115 & 4.4 & 1110 & 23.8 \\
\hline $\mathrm{C} 14$ & 58 & 57 & 79 & 1620 & 1680 & 1680 & 198.9 & 1710 & 6.4 & 1680 & 54.6 \\
\hline $\mathrm{C} 15$ & 97 & 107 & 140 & 1765 & 1860 & 1860 & 552.3 & 1910 & 30.8 & 1865 & 335.3 \\
\hline C16 & 32 & 32 & 42 & 580 & 585 & 585 & 122.9 & 585 & 0.8 & 585 & 5.1 \\
\hline $\mathrm{C} 17$ & 43 & 42 & 56 & 1590 & 1610 & 1610 & 137.5 & 1630 & 3.2 & 1610 & 14.0 \\
\hline C18 & 93 & 121 & 133 & 2315 & 2410 & 2410 & 565.6 & 2460 & 38.8 & 2415 & 520.8 \\
\hline C19 & 62 & 61 & 84 & 1345 & 1395 & 1395 & 210.2 & 1425 & 7.0 & 1400 & 76.2 \\
\hline $\mathrm{C} 20$ & 45 & 53 & 64 & 665 & 665 & 665 & 1.2 & 795 & 1.0 & 665 & 2.6 \\
\hline $\mathrm{C} 21$ & 60 & 76 & 84 & 1705 & 1725 & 1725 & 326.9 & 1725 & 3.7 & 1725 & 32.0 \\
\hline $\mathrm{C} 22$ & 56 & 43 & 76 & 1070 & 1070 & 1070 & 2.8 & 1070 & 0.1 & 1070 & 0.7 \\
\hline $\mathrm{C} 23$ & 78 & 92 & 109 & 1620 & 1700 & 1690 & 381.6 & 1775 & 18.9 & 1700 & 99.5 \\
\hline $\mathrm{C} 24$ & 77 & 84 & 115 & 1330 & 1360 & 1360 & 311.4 & 1405 & 6.0 & 1360 & 78.9 \\
\hline $\mathrm{C} 25$ & 37 & 38 & 50 & 905 & 905 & 905 & 0.3 & 935 & 1.9 & 905 & 0.7 \\
\hline \multicolumn{4}{|c|}{ Average } & 1449.8 & 1500.0 & 1500.8 & 236.0 & 1555.2 & 9.5 & 1501.0 & 85.85 \\
\hline \multicolumn{4}{|c|}{ No. optimal } & - & 4 & 4 & - & 1 & - & 4 & - \\
\hline \multicolumn{4}{|c|}{$\begin{array}{l}\text { Average deviation } \\
\text { to the LB }(\%)\end{array}$} & - & 3.46 & 3.52 & - & 7.27 & - & 3.53 & - \\
\hline
\end{tabular}

Optimal solutions are in bold.

New best solutions are in italic.

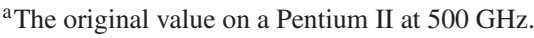

application in Lancashire [22-24]. There are 24 instances based on two graphs where the different instances have been created by changing the set of required edges and the capacities of the vehicles. Because of the application from which these instances were generated, the demand quantities for the required edges are proportional to their costs.

The full data for these instances can be obtained from http://www.uv.es/ belengue/carp.html.

To give an impression of the relative accuracy of the five initial solution methods, the average solution value was calculated for each method over all the instances in each set and this was compared to the average best known solution from the literature over all the instances in each set. The ratios of these averages are given in Table 1 . It will be seen that the quality of these initial solutions is poor compared to the final solutions generated by the TSA.

The results are presented in Tables 2-4. For each instance, an indication of the size of the graph is given, where $n$ is the number of vertices, $N$ is the number of required edges and $M$ is the total number of edges. The tables show the best known results from the literature. The column headed "Our best" gives the result from all runs of our algorithm that included trials of different swap frequencies. The columns headed "CARPET" give the results reported in Hertz et al. [7]. The run times have been divided by 7 to give an approximate comparison between the times on the original computer used by the authors (SGI Indigo2 at $195 \mathrm{MHz}$ ) and our algorithm that was coded in $\mathrm{C}$ and run using a Pentium Mobile at 1.4 GHz. The columns headed "MA" give the results reported by Lacomme et al. [8], using a Pentium III at $1 \mathrm{GHz}$. The computing times in their paper have been divided by 1.5 to make them approximately equivalent to 
Table 6

Problems from Beullens et al.—set D

\begin{tabular}{|c|c|c|c|c|c|c|c|c|c|c|c|}
\hline \multirow[t]{2}{*}{ Name } & \multirow[t]{2}{*}{$|V|$} & \multirow[t]{2}{*}{$|R|$} & \multirow[t]{2}{*}{$|E|$} & \multirow[t]{2}{*}{ LB } & \multirow[t]{2}{*}{ Our best } & \multicolumn{2}{|c|}{ Beullens et al. } & \multicolumn{2}{|c|}{ TSA Version 1} & \multicolumn{2}{|c|}{ TSA Version 2} \\
\hline & & & & & & Cost & CPU (s) & Cost & $\mathrm{CPU}(\mathrm{s})$ & Cost & $\mathrm{CPU}(\mathrm{s})$ \\
\hline D1 & 69 & 79 & 98 & 725 & 740 & 725 & 11.8 & 865 & 2.8 & 740 & 62.7 \\
\hline D2 & 48 & 53 & 66 & 480 & 480 & 480 & 1.2 & 480 & 1.6 & 480 & 0.8 \\
\hline D3 & 46 & 51 & 64 & 415 & 415 & 415 & 0.4 & 415 & 0.2 & 415 & 0.2 \\
\hline D4 & 60 & 72 & 84 & 615 & 615 & 615 & 0.5 & 630 & 4.4 & 615 & 1.7 \\
\hline D5 & 56 & 65 & 79 & 1040 & 1040 & 1040 & 1.4 & 1080 & 2.4 & 1040 & 2.8 \\
\hline D6 & 38 & 51 & 55 & 485 & 485 & 485 & 0.2 & 525 & 0.7 & 485 & 7.9 \\
\hline D7 & 54 & 52 & 70 & 735 & 835 & 835 & 138.9 & 915 & 3.4 & 835 & 22.8 \\
\hline D8 & 66 & 63 & 88 & 615 & 685 & 685 & 195.1 & 685 & 4.5 & 685 & 49.1 \\
\hline D9 & 76 & 97 & 117 & 680 & 680 & 680 & 1.1 & 810 & 6.1 & 680 & 30.1 \\
\hline D10 & 60 & 55 & 82 & 900 & 910 & 910 & 149.1 & 910 & 1.3 & 910 & 16.5 \\
\hline D11 & 83 & 94 & 118 & 920 & 940 & 930 & 368.3 & 990 & 8.0 & 960 & 112.0 \\
\hline D12 & 62 & 72 & 88 & 680 & 680 & 680 & 0.4 & 735 & 2.5 & 680 & 26.0 \\
\hline D13 & 40 & 52 & 60 & 690 & 690 & 690 & 0.8 & 695 & 1.1 & 695 & 13.9 \\
\hline D14 & 58 & 57 & 79 & 920 & 930 & 930 & 180.2 & 950 & 1.0 & 940 & 21.1 \\
\hline D15 & 97 & 107 & 140 & 910 & 950 & 910 & 368.8 & 1100 & 23.6 & 950 & 102.3 \\
\hline D16 & 32 & 32 & 42 & 170 & 170 & 170 & 0.0 & 175 & 0.1 & 170 & 0.4 \\
\hline D17 & 43 & 42 & 56 & 675 & 675 & 675 & 0.2 & 675 & 0.0 & 675 & 0.0 \\
\hline D18 & 93 & 121 & 133 & 930 & 930 & 930 & 2.5 & 1075 & 13.9 & 930 & 190.5 \\
\hline D19 & 62 & 61 & 84 & 650 & 680 & 680 & 154.8 & 690 & 2.7 & 690 & 20.1 \\
\hline D20 & 45 & 53 & 64 & 415 & 415 & 415 & 0.1 & 420 & 2.5 & 415 & 1.1 \\
\hline D21 & 60 & 76 & 84 & 695 & 815 & 805 & 251.8 & 865 & 2.3 & 825 & 60.9 \\
\hline D22 & 56 & 43 & 76 & 690 & 690 & 690 & 0.3 & 690 & 0.0 & 690 & 0.0 \\
\hline D23 & 78 & 92 & 109 & 715 & 735 & 735 & 335.3 & 780 & 5.3 & 735 & 111.1 \\
\hline D24 & 77 & 84 & 115 & 620 & 670 & 670 & 248.0 & 670 & 10.3 & 670 & 105.2 \\
\hline D25 & 37 & 38 & 50 & 410 & 410 & 410 & 0.0 & 510 & 0.2 & 410 & 0.6 \\
\hline \multicolumn{3}{|c|}{ Average } & & 671.2 & 690.6 & 687.6 & 96.4 & 733.4 & 4.0 & 693.2 & 38.4 \\
\hline \multicolumn{3}{|c|}{ No. optimal } & & & 14 & 15 & & 4 & - & 13 & - \\
\hline \multicolumn{2}{|c|}{$\begin{array}{l}\text { Average deviation } \\
\text { to the LB }(\%)\end{array}$} & & & - & 2.89 & 2.44 & & 9.27 & - & 3.28 & - \\
\hline
\end{tabular}

Optimal solutions are in bold.

the times we recorded for our algorithm. The best results include those reported by Beullens et al. [9], Baldacci and Maniezzo [13] and Longo et al. [14].

In Tables 2 and 3, if the best known or our best solution is optimal then it is shown in bold. Optimality can be proved for many of the problems in the first two sets using the lower bounding procedures described in Belenguer and Benavent [25]. However, these lower bounding procedures still leave a gap between the lower bound and the best known solutions for the larger problems in the third set. Ahr [26], Baldacci and Maniezzo [13] and Longo et al. [14] have found improvements to some of these lower bounds and the column LB in Table 4 gives the best lower bounds found so far for these problems. Note that the lower bounds reported for the second set of test problems in Belenguer and Benavent [25] differ from those reported in Hertz et al. [7] and Lacomme et al. [8]. This is due to a different cost being used for servicing the required edges. As this is a fixed cost incurred by any solution, the consequence is just that a constant term is needed to adjust the results. Details of the adjustments needed are provided in Belenguer and Benavent [25].

The results show that the TSA is capable of providing high quality results. Using the best results from all trials of the TSA with varying swap frequencies and running times, "our best" solutions match the best solutions found for all instances in the first set, all instances except the last one in the second set and in the third set of large instances, "our best" solutions are at least as good as the best published results in all 24 instances and in 17 of these instances, a new best solution has been found using the TSA.

However in order to properly evaluate the TSA, we should examine the results of Version 1 and Version 2 where the parameters have been fixed. In our computational experiments, we have followed the practice of other researchers by 
Table 7

Problems from Beullens et al.—set E

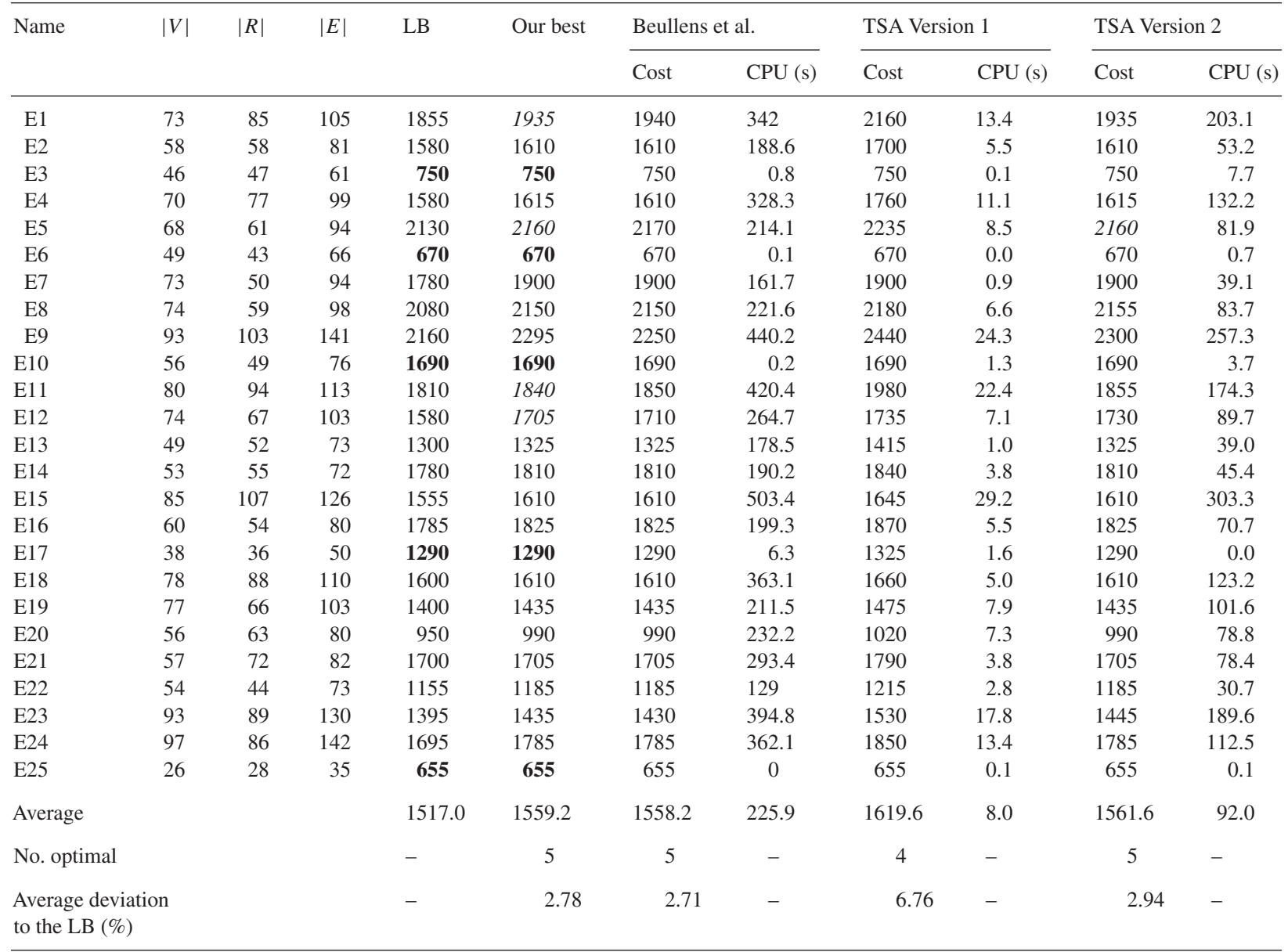

Optimal solutions are in bold.

New best solutions are in italic.

halting the programme when a known optimal solution has been found. As expected, Version 1 is faster and the average solution over all the instances in each set only exceeds the best known solution or lower bound on the optimal solution (for the third set) by $0.55 \%, 0.90 \%$ and $2.33 \%$, respectively, for the three problem sets. Version 2 requires longer, but the average solution over all the instances in each set exceeds the best known solution or lower bound on the optimal solution (for the third set) by $0.08 \%, 0.15 \%$ and $1.54 \%$, respectively.

Comparison can be made with CARPET for the first two sets of problems. This shows that the average solution values given by CARPET and Version 1 are very similar, but Version 1 is much faster over all the problems. Version 2 gives slightly better solutions on average than CARPET, finds more best solutions and is still significantly faster.

Comparison can be made with MA for all three sets of problems. Version 1 is much faster than MA, but the results are not quite so good. Version 2 gives results that are slightly better and the computing time is lower than required for MA.

Further experiments were conducted on the 100 CARP data sets generated by Beullens et al. [9] based on the intercity road network in Flanders (Belgium). Tables 5-8 show the results for the four sets of instances, C, D, E and F. Sets $\mathrm{D}$ and $\mathrm{F}$ are based on the same networks, but using vehicles of larger capacity. As in Beullens et al. [9], the results are reported in terms of the cost of deadheading only. The results show that the TSA is competitive with the guided local search heuristic proposed by Beullens et al. [9]. Over the 100 problems, the TSA equalled the best solution found by the guided local search heuristic for 78 instances and additionally found seven new best solutions. 
Table 8

Problems from Beullens et al.- - set F

\begin{tabular}{|c|c|c|c|c|c|c|c|c|c|c|c|}
\hline \multirow[t]{2}{*}{ Name } & \multirow[t]{2}{*}{$|V|$} & \multirow[t]{2}{*}{$|R|$} & \multirow[t]{2}{*}{$|E|$} & \multirow[t]{2}{*}{ LB } & \multirow[t]{2}{*}{ Our best } & \multicolumn{2}{|c|}{ Beullens et al. } & \multicolumn{2}{|c|}{ TSA Version 1} & \multicolumn{2}{|c|}{ TSA Version 2} \\
\hline & & & & & & Cost & CPU (s) & Cost & CPU (s) & Cost & $\mathrm{CPU}(\mathrm{s})$ \\
\hline $\mathrm{F} 1$ & 73 & 85 & 105 & 1065 & 1070 & 1065 & 3.4 & 1090 & 11.5 & 1085 & 88.5 \\
\hline $\mathrm{F} 2$ & 58 & 58 & 81 & 920 & 920 & 920 & 2.3 & 940 & 2.6 & 920 & 0.3 \\
\hline F3 & 46 & 47 & 61 & 400 & 400 & 400 & 0.0 & 480 & 1.8 & 400 & 1.3 \\
\hline $\mathrm{F} 4$ & 70 & 77 & 99 & 930 & 945 & 940 & 247.8 & 970 & 2.7 & 960 & 63.7 \\
\hline F5 & 68 & 61 & 94 & 1180 & 1180 & 1180 & 0.2 & 1185 & 1.8 & 1180 & 17.1 \\
\hline F6 & 49 & 43 & 66 & 490 & 490 & 490 & 0.0 & 540 & 0.4 & 490 & 0.0 \\
\hline F7 & 73 & 50 & 94 & 1080 & 1080 & 1080 & 0.1 & 1110 & 3.6 & 1080 & 1.7 \\
\hline F8 & 74 & 59 & 98 & 1135 & 1145 & 1145 & 185.5 & 1155 & 1.1 & 1145 & 35.7 \\
\hline F9 & 93 & 103 & 141 & 1145 & 1155 & 1145 & 3.7 & 1520 & 7.4 & 1170 & 145.9 \\
\hline F10 & 56 & 49 & 76 & 1010 & 1010 & 1010 & 0.1 & 1010 & 0.1 & 1010 & 0.1 \\
\hline F11 & 80 & 94 & 113 & 1015 & 1015 & 1015 & 4.5 & 1100 & 8.5 & 1015 & 125.8 \\
\hline F12 & 74 & 67 & 103 & 900 & 910 & 910 & 228.7 & 1000 & 4.9 & 910 & 47.9 \\
\hline $\mathrm{F} 13$ & 49 & 52 & 73 & 835 & 835 & 835 & 0.2 & 855 & 0.8 & 835 & 0.2 \\
\hline F14 & 53 & 55 & 72 & 1025 & 1035 & 1025 & 18.8 & 1085 & 2.5 & 1035 & 29.7 \\
\hline F15 & 85 & 107 & 126 & 945 & 965 & 945 & 1.3 & 1315 & 22.1 & 990 & 145.4 \\
\hline F16 & 60 & 54 & 80 & 775 & 775 & 775 & 0.1 & 945 & 1.9 & 775 & 3.4 \\
\hline F17 & 38 & 36 & 50 & 605 & 605 & 605 & 0.1 & 660 & 0.3 & 630 & 4.7 \\
\hline F18 & 78 & 88 & 110 & 835 & 850 & 850 & 274.5 & 945 & 13.9 & 850 & 92.9 \\
\hline F19 & 77 & 66 & 103 & 685 & 725 & 725 & 158.0 & 740 & 1.5 & 740 & 34.9 \\
\hline F20 & 56 & 63 & 80 & 610 & 610 & 610 & 1.3 & 610 & 0.3 & 610 & 9.8 \\
\hline F21 & 57 & 72 & 82 & 905 & 905 & 905 & 4.0 & 940 & 2.1 & 905 & 45.5 \\
\hline F22 & 54 & 44 & 73 & 790 & 790 & 790 & 0.4 & 790 & 0.1 & 790 & 0.4 \\
\hline F23 & 93 & 89 & 130 & 705 & 730 & 725 & 319.6 & 895 & 4.6 & 730 & 80.2 \\
\hline F24 & 97 & 86 & 142 & 975 & 1010 & 975 & 26.6 & 1040 & 10.3 & 1010 & 79.2 \\
\hline F25 & 26 & 28 & 35 & 430 & 430 & 430 & 0.1 & 430 & 0.0 & 430 & 0.1 \\
\hline \multicolumn{4}{|c|}{ Average } & 855.6 & 863.4 & 859.8 & 59.3 & 934.0 & 4.3 & 867.8 & 42.2 \\
\hline \multicolumn{4}{|c|}{ No. optimal } & - & 14 & 19 & - & 4 & - & 13 & - \\
\hline \multicolumn{2}{|c|}{$\begin{array}{l}\text { Average deviation } \\
\text { to the LB }(\%)\end{array}$} & & & - & 0.91 & 0.49 & - & 9.16 & $\begin{array}{l}- \\
-\end{array}$ & 1.43 & - \\
\hline
\end{tabular}

Optimal solutions are in bold.

New best solutions are in italic.

Table 9

Large problems

\begin{tabular}{|c|c|c|c|c|c|c|}
\hline \multirow[t]{2}{*}{ Name } & \multirow[t]{2}{*}{$|V|$} & \multirow[t]{2}{*}{$|R|$} & \multirow[t]{2}{*}{$|E|$} & \multirow{2}{*}{$\begin{array}{l}\text { Initial solution cost } \\
\text { (path scanning method) }\end{array}$} & \multicolumn{2}{|c|}{ TSA Version 1} \\
\hline & & & & & Cost & CPU (s) \\
\hline G1-A & 255 & 347 & 375 & 1318092 & 1049708 & 789.4 \\
\hline G1-B & 255 & 347 & 375 & 1483179 & 1140692 & 867.3 \\
\hline G1-C & 255 & 347 & 375 & 1584177 & 1282270 & 919.1 \\
\hline G1-D & 255 & 347 & 375 & 1744159 & 1420126 & 850.8 \\
\hline G1-E & 255 & 347 & 375 & 1841023 & 1583133 & 672.2 \\
\hline G2-A & 255 & 375 & 375 & 1416720 & 1129229 & 1455.6 \\
\hline G2-B & 255 & 375 & 375 & 1559464 & 1255907 & 1122.3 \\
\hline G2-C & 255 & 375 & 375 & 1704234 & 1418145 & 849.0 \\
\hline G2-D & 255 & 375 & 375 & 1918757 & 1516103 & 1805.3 \\
\hline G2-E & 255 & 375 & 375 & 1998355 & 1701681 & 879.9 \\
\hline Average & & & & 1656816.0 & 1349699.4 & 1021.1 \\
\hline
\end{tabular}


Finally a new set of large instances was created for testing. These were based on a different road network in Lancashire that had been used in a winter gritting study with 255 vertices and 375 edges. Different problem instances were created by changing the set of edges required for service and by changing the capacity of the vehicles. Table 9 shows the results from using one of the classical heuristics, the path scanning method, and comparing it with the solution provided by running Version 1 of the TSA. The path scanning method is fast, taking an average of only $0.27 \mathrm{~s}$ to find a solution. However the TSA improved the results from the path scanning method by $18.5 \%$, taking an average time of $1021.1 \mathrm{~s}$.

\section{Conclusions}

The paper has demonstrated that the TSA is able to provide high quality solutions to the capacitated arc routing problem (CARP) in a reasonable computing time. Several new best solutions are provided for the Eglese set of test problems that have been studied by other researchers.

The results presented demonstrate the good performance of the TSA compared to CARPET and the memetic algorithms approach (MA) of Lacomme et al. [8]. In addition, the TSA is a deterministic algorithm, so all the results are fully reproducible. Both CARPET and MA include several random elements, so different runs of these algorithms may produce different results. CARPET is also complex in the subroutines used within the algorithm. MA is also a complex algorithm and although it has the potential to be easily extended to other problems, it requires many parameters to be set.

The guided local search approach of Beullens et al. [9] describes an alternative deterministic algorithm for solving the CARP. Their approach also provides high quality solutions in a limited computation time. The results show that the TSA is competitive with their approach and has found some new best solutions for the problem instances they introduced.

This paper demonstrates that a relatively straightforward implementation of tabu search (without any long-term memory component or any other procedures to encourage diversification apart from different starting solutions and different frequencies for different types of move) is able to produce high quality solutions to the CARP in an efficient manner.

\section{References}

[1] Dror M. (Ed.). Arc routing. Theory, solutions and applications. Boston: Kluwer Academic Publishers; 2000.

[2] Lacomme P, Prins C, Ramdane-Cherif W. Evolutionary algorithms for periodic arc routing problems. European Journal of Operational Research $2005 ; 165: 535-53$.

[3] Lenstra JK, Rinnooy Kan AHG. On general routing problems. Networks 1976;6:273-80.

[4] Golden BL, Wong RT. Capacitated arc routing problems. Networks 1981;11:305-15.

[5] Pearn WL. Approximate solutions for the capacitated arc routing problem. Computers and Operations Research 1989;16(6):589-600.

[6] Pearn WL. Augment-insert algorithms for the capacitated arc routing problem. Computers and Operations Research 1991;18(2):189-98.

[7] Hertz A, Laporte G, Mittaz M. A tabu search heuristic for the capacitated arc routing problem. Operations Research 2000;48(1):129-35.

[8] Lacomme P, Prins C, Ramdane-Cherif W. Competitive memetic algorithms for arc routing problems. Annals of Operational Research 2004;131(1-4):159-85.

[9] Beullens P, Muyldermans L, Cattrysse D, Van Oudheusden D. A guided local search heuristic for the capacitated arc routing problem. European Journal of Operational Research 2003; 147:629-43.

[10] Greistorfer P. A tabu search metaheuristic for the arc routing problem. Preprint. Amsterdam: Elsevier Science; 2002.

[11] Hertz A, Mittaz M. A variable neighborhood descent algorithm for the undirected capacitated arc routing problem. Transportation Science 2001;35(4):425-34.

[12] Amberg A, Domschke W, Voss S. Multiple center capacitated arc routing problems: a tabu search algorithm using capacitated trees. European Journal of Operational Research 2000;124:360-76.

[13] Baldacci R, Maniezzo V. Exact methods based on node-routing formulations for undirected arc-routing problems. Networks 2006;47:52-60.

[14] Longo H, Poggi de Aragão M, Uchoa E. Solving capacitated arc routing problems using a transformation to the CVRP. Computers and Operations Research 2006;33:1823-37.

[15] Edmonds J, Johnson EL. Matching, Euler tours and the Chinese postman problem. Mathematical Programming 1973;5:88-124.

[16] Frederickson GN. Approximation algorithms for some postman problems. Journal of the ACM 1979;26(3):538-54.

[17] Christofides N. Worst-case analysis of a new heuristic for the traveling salesman problem. Report No. 388, Graduate School of Industrial Administration, Carnegie Mellon University, Pittsburgh; 1976.

[18] Pearn WL, Wu TC. Algorithms for the rural postman problem. Computers and Operations Research 1995;22(8):819-28.

[19] Golden BL, DeArmon JS, Baker EK. Computational experiments with algorithms for a class of routing problems. Computers and Operations Research 1983;10(1):47-59. 
[20] DeArmon JS. A comparison of heuristics for the capacitated Chinese postman problem. Master's thesis, University of Maryland, College Park, MD; 1981.

[21] Benavent E, Campos V, Corberán E, Mota E. The capacitated arc routing problem. Lower bounds. Networks 1992;22:669-90.

[22] Eglese RW. Routing winter gritting vehicles. Discrete Applied Mathematics 1994;48(3):231-44.

[23] Eglese RW, Li LYO. A tabu search based heuristic for arc routing with a capacity constraint and time deadline. In: Osman IH, Kelly JP, editors. Metaheuristics: theory and applications. Boston: Kluwer Academic Publishers; 1996. p. 633-50.

[24] Li LYO, Eglese RW. An interactive algorithm for vehicle routing for winter-gritting. Journal of the Operational Research Society 1996;47: 217-28.

[25] Belenguer JM, Benavent E. A cutting plane algorithm for the capacitated arc routing problem. Computers and Operations Research 2003;30(5):705-28.

[26] Ahr D. Contributions to multiple postmen problems. Ph.D. Dissertation, Ruprecht-Karls-Universität, Heidelberg; 2004. 